\title{
Candidiasis as Secondary Infection in Post COVID-19: A New Problem?
}

\author{
Evelyn Nathania $^{1 \wedge}{ }^{(1 D}$; Jahja Teguh Widjaja ${ }^{2}$ \\ ${ }^{1}$ Immanuel Hospital, Bandung, Indonesia. \\ ${ }^{2}$ Department of Pulmonology and Respiratory Medicine, Immanuel Hospital, Bandung, Indonesia.
}

\section{ARTICLE INFO}

\section{Article history:}

Received 23 April 2021

Received in revised form 21

September 2021

Accepted 24 September 2021

Available online 30 September 2021

\section{Keywords:}

Candidiasis,

COVID-19,

Fungal pneumonia,

Infectious disease,

Secondary infection.

\begin{abstract}
Introduction: Severe Acute Respiratory Syndrome (SARS-CoV-2) can develop into post COVID-19 with one of its main attentions being secondary infection. Candidiasis as fungal pneumonia is one of secondary infection in post COVID-19. It is hard to diagnose and has high mortality. We present a case of candidiasis as fungal pneumonia in post COVID-19 patient.

Case: A 47-year-old woman came to the emergency room with major complaints of dyspnoea and jaundice since her second day at home after being discharged from having COVID-19. She previously finished her COVID treatment and tested negative 2 times. She had severe sepsis and her sputum culture was positive for non-albicans Candida. She received anti-fungal treatment and her condition was getting better and her chest Xray showed less infiltrates than before.

Discussion: Fungal respiratory infections generate concern in the expanding population of immunosuppressed patients like COVID-19 patient. The mortality from COVID-19 associated candidiasis is high (19-40\%) as one of fungal infection. Marked immune dysregulation in COVID-19 makes system immune becomes impaired, it also harms lung epithelium. In this patient, diagnosis and treatment were challenging.

Conclusion: Post COVID-19 is a condition that should be given more attention, especially when it develops secondary infection. Candidiasis as fungal pneumonia is hard to diagnose and can quickly become severe sepsis and has high mortality. Health practitioners should recognize it as soon as possible and give the best treatment for the patient.
\end{abstract}

\section{INTRODUCTION}

Severe Acute Respiratory Syndrome (SARS$\mathrm{CoV}-2)$ is an illness caused by novel coronavirus (COVID-19). World Health Organization (WHO) declared it as global pandemic in March $2020 .{ }^{1}$ It has infected around 215 countries and more than 31 million people. Even after being treated or after being negative in PCR tests, it can develop into long COVID or secondary infections and even multiorgan dysfunction. ${ }^{2}$ Viral pneumonia, especially COVID-19, carries a risk of developing second infection. Fungal infections are common complication. Nevertheless, most of it are usually being underdiagnosed or being late diagnosis, while the mortality in pneumonia because of candida reaches $19-40 \% .^{3}$ It can also quickly develop in severe sepsis and can worsen patient's condition. We report a case of a 47-year-old woman with post COVID-19 who came to the emergency room with major complaints of jaundice and dyspnoea with hypotension. Her chest Xray showed infiltrates, later on her sputum culture was positive with non-albicans Candida. She was diagnosed with candidiasis as fungal pneumonia as secondary infection in post COVID-19.

\section{CASE}

A 47-year-old woman came to the emergency room with major complaints of dyspnoea and jaundice from two days ago. A day before her complaints, she was discharged from the hospital for having moderate

*Corresponding author: nathania_evelynlogamarta@gmail.com 
COVID-19 infection. She previously completed her COVID-19 treatment for 14 days and being tested negative two times for PCR swab tests with no symptoms at all before going home. She had no other diseases such as diabetes mellitus, hypertension, congestive heart failure, and cholangitis or hepatitis. On admission, her blood pressure was 90/60, her temperature was $36.6^{\circ}$ Celsius, her heart rate was $137 \mathrm{x} /$ minute, and her respiratory rate was $32 \mathrm{x} /$ minute with $97 \% \mathrm{SpO}_{2}$ without oxygen support. Her sclera and her skin were icteric without organomegaly. She had no JVP elevation, no ronchi on auscultation, and no oedema. Her chest X-ray showed bilateral infiltrates with cardiomegaly (Figure 3 ). Her laboratory findings showed anaemia with thrombocytopenia and leucocytosis. Her SGOT and SGPT were risen with hyponatremia (Table 1). She was given digoxin, spironolactone, antibiotic, vitamin for liver, and furosemide. She was suspected of Acute Decompensated Heart Failure (ADHF) with congestive liver as complication. Her differential diagnosis were sepsis and bronchopneumonia with unknown underlying disease in post COVID-19. Her ECG was normal.

On the second day of admission, her dyspnoea was getting worse, her vital sign dropped, her tension was 70/50, her condition was getting worse and she was given norepinephrine with dobutamine. She did not get any better with her previous treatment, even with furosemide. We evaluated her heart condition and her echocardiography (Figure 1) was normal with $76 \%$ ejection fraction (heart chamber dimension was normal, systolic function was normal, and diastolic dysfunction only shown grade I, normo-kinetic). Her liver and bile duct USG (Figure 2) were also normal and showed no stasis, she showed no cholelithiasis and she was negative for hepatitis A, B and C parameters. Even after receiving broad spectrum antibiotics, her condition was getting worse. She had no response for antibiotics. She showed no oral candidiasis or leucoplakia. After reviewing her condition, we suggested to do a bronchoscopy to take a sample for culture, but her condition was unstable and her family did not agree for bronchoscopy; therefore we only took the sample from her sputum. On her fifth day of admission, her sputum culture showed non-albicans Candida. Based on sputum culture, clinical condition, and chest X-ray, she was given echinocandins as antifungal, and her condition was getting better. On the following day, her haemoglobin was only 8.1. 5 days later it dropped again to 6.8, her CRP increased and she had hypoalbuminemia and was high on D-dimer. We gave her PRC transfusion and continued her anti-fungal treatment. She achieved stability three days later and felt better afterwards. On her $10^{\text {th }}$ day in the hospital, she felt no fatigue, showing no dyspnoea, and her jaundice was getting better. At the end of her anti-fungal treatment, her thorax showed less infiltrates than before (Figure 4) and she had no complaints. She was discharged from the hospital and came to polyclinic for follow-up treatment after one week. She had no complaints, no dyspnoea, no jaundice, and regained a better quality of life than before. Her thorax also showed less infiltrate (Figure 5).

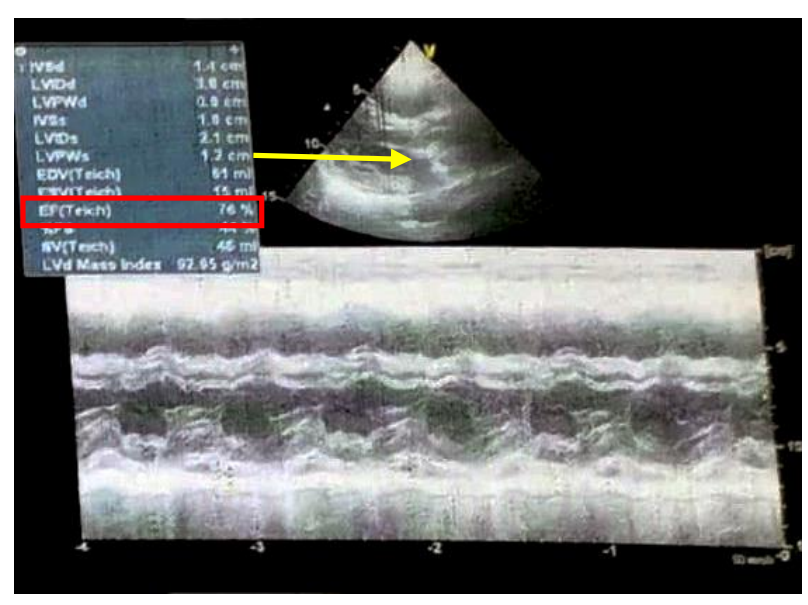

Figure 1. Echocardiography showed normal chamber (yellow arrow) with normal EF (red square) and systolic function

Table 1. Laboratory findings

\begin{tabular}{|c|c|c|c|c|c|c|c|}
\hline Laboratory Findings & $\begin{array}{l}\text { Reference } \\
\text { Range }\end{array}$ & Admis & Day 5 & Day 6 & Day 11 & Day 12 & Day 13 \\
\hline $\mathrm{Hb}(\mathrm{g} / \mathrm{dL})$ & $11.7-15.5$ & 10.6 & - & 8.1 & - & 6.8 & 11.1 \\
\hline Leucocytes $\left(10^{3} / \mathrm{mm}^{3}\right)$ & $4.00-10.00$ & 16.06 & - & 15.65 & - & 19.43 & 12.66 \\
\hline Platelet Count $\left(10^{9} / \mathrm{L}\right)$ & $150-450$ & 94 & & 221 & - & 496 & 642 \\
\hline Natrium (mEg/dL) & $136-146$ & 123 & - & 131 & - & 135 & - \\
\hline D-Dimer $(\mu \mathrm{g} / \mathrm{mL})$ & $<0.5$ & - & 4 & - & 1.72 & - & - \\
\hline Albumin (g/dL) & $3.8-5.5$ & - & - & 2.8 & - & - & - \\
\hline Fibrinogen (mg/L) & $200-400$ & - & - & - & - & - & 487 \\
\hline SGOT (U/L) & $<31$ & 140 & - & 57 & - & 41 & - \\
\hline SGPT (U/L) & $<31$ & 116 & - & 55 & - & 36 & - \\
\hline Total Bilirubin (mg/dL) & $<0.1$ & - & - & 5.53 & - & 2.28 & - \\
\hline Direct Bilirubin (mg/dL) & $<0.4$ & - & - & 4.02 & - & 2.08 & - \\
\hline Indirect Bilirubin (mg/dL) & $<0.6$ & - & - & 1.51 & - & 0.77 & - \\
\hline CRP Quantitative (mg/L) & $<10$ & - & - & - & - & 36.74 & - \\
\hline Procalcitonin & $<0.5$ & - & 0.15 & - & - & - & - \\
\hline
\end{tabular}




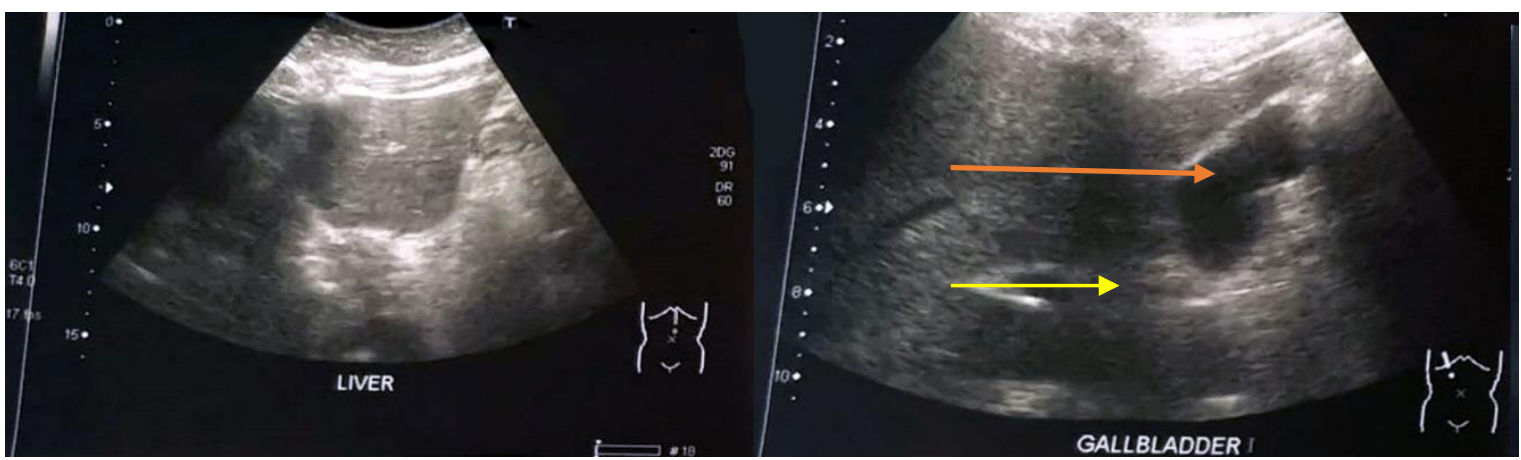

Figure 2. Abdomen USG showed normal liver (blue arrow), normal gallbladder (yellow arrow), and normal CBD (green arrow)

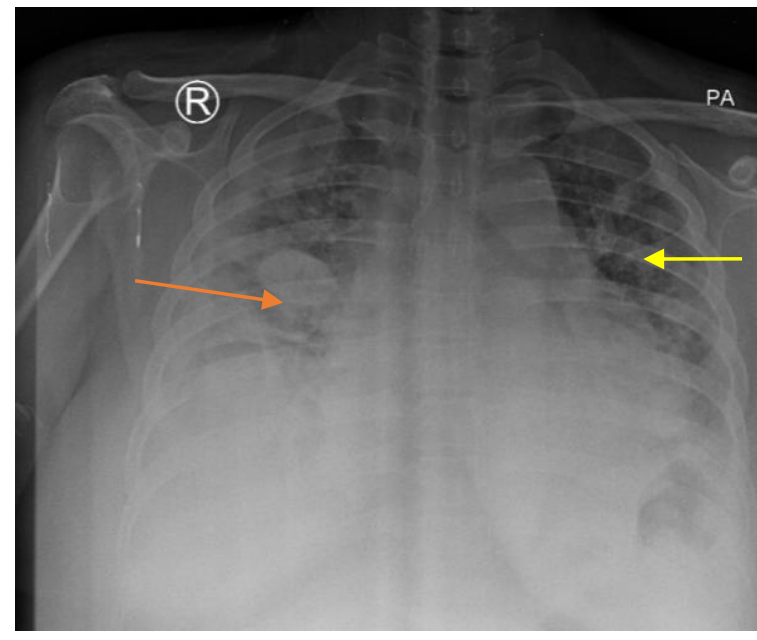

Figure 3. Day 0 admission thorax, full infiltrate (yellow arrow)

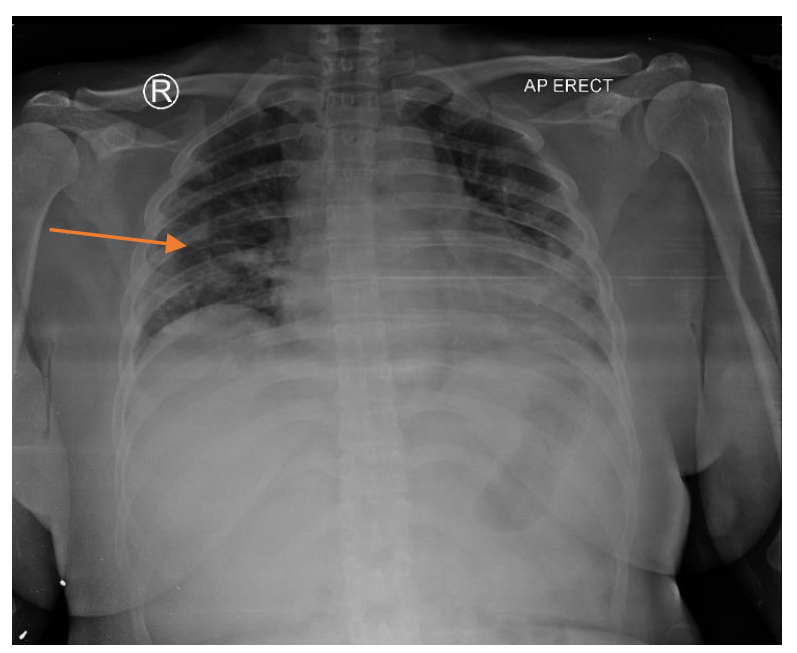

Figure 4. After receiving anti-fungal thorax, less infiltrate (yellow arrow)

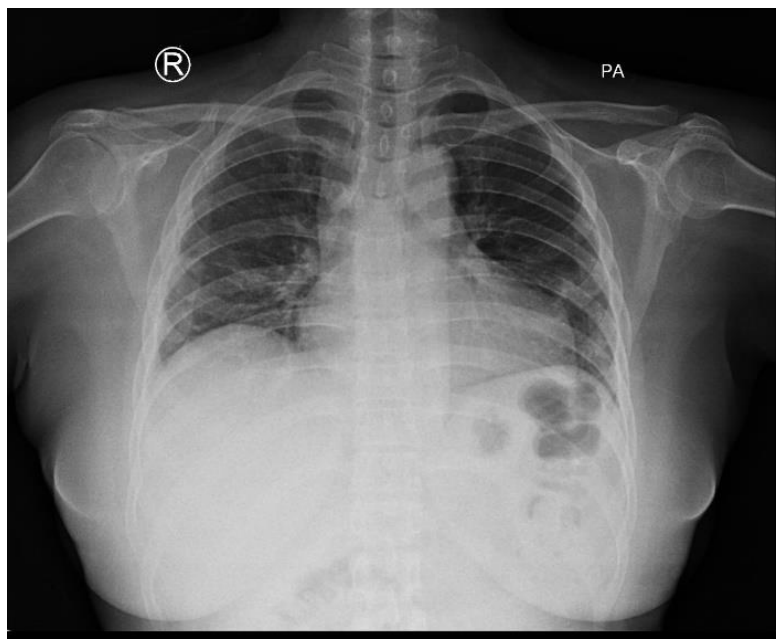

Figure 5. Follow-up in polyclinic after one week, less infiltrate (yellow arrow)

\section{DISCUSSION}

COVID-19 is caused by SARS-CoV-2 and has been the source of pandemic since 2020. Many people suffered on many aspects of life, especially health issues. However, a new problem occurred. People are suffering from symptoms after SARS-CoV-2 infection and the number is increasing. ${ }^{4}$ Post COVID-19 is a condition that persists for more than four months after being infected with the virus. Most people with COVID19 got better within weeks to months of illness, but some did not. ${ }^{2}$ The mortality and morbidity associated with COVID-19 are caused by the response of host immune. ${ }^{5}$

The pathomechanism infection differs from other viruses like chronic HIV or HCV infection. Prolonged SARS-CoV-2 infection exposure leads to excessive $\mathrm{T}$ cell activation and immune responses, in which terminal 
differentiation into effector cells predominates memory cell development. This can be followed by cell exhaustion, resulting in lymphopenia and can affects patient's condition. ${ }^{6}$ Concomitantly, $\mathrm{T}$ cells expressed high levels of PD-1, which is involved in $\mathrm{T}$ cell inhibition. Elevated PD-1 expression on CD4+ and $\mathrm{CD} 8+\mathrm{T}$ cells is found on activated $\mathrm{T}$ cells and has been recognized as an indicator for cell exhaustion during chronic human viral infections and in COVID-19. Thus, it may be relevant for the outcome of COVID-19 and increasing its mortality and morbidity. ${ }^{7}$ Post COVID-19 is hard to treat, especially those which developed into secondary infection and multiorgan dysfunction. Just like other viral pneumonias, bacterial and fungal infections can be seen as complications in hospitalized COVID-19 patients. $^{8}$ Secondary infection in post COVID-19 is a new problem that should be recognized and treated early. We report a case of candidiasis as secondary infection in post COVID-19 patient.

Fungal respiratory infections generate concern in the expanding population of immunosuppressed patients like COVID-19 patient. Fungi may colonize body sites without producing disease or they may be a true pathogen, generating a broad variety of clinical syndromes. Fungal pneumonia is an infectious process in the lungs caused by one or more endemic or opportunistic fungi. Opportunistic fungal organisms (Candida species, Aspergillus species, Mucor species) tend to cause pneumonia in patients with congenital or acquired defects in the host immune defences. ${ }^{9}$

Candidiasis as fungal pneumonia is hard to diagnose and often underestimated, meanwhile the mortality from COVID-19 associated candidiasis is high $(19-40 \%) .^{3} 3$ out of 12 patients in Wuhan, China, who were being tested for sputum culture, had positive for fungal culture instead of bacterial culture. ${ }^{10}$ As in India, out of a total of 7,163 samples collected for microbiological culture, $852(11.89 \%)$ samples were positive for bacterial or fungal growth. Candida spp. were isolated from $6 \%$ (61/1006) of admitted COVID19 patients, and, among these, 13 isolates (1.3\%) of Candida auris were found. ${ }^{8}$ Infections continue to develop in hospitalized COVID-19 patients. ${ }^{11}$ COVID19 carries a risk of developing second infection, and Candida is the main cause of fungal infection with high mortality rate. Main fungal pathogens in COVID-19 are Aspergillus and Candida, but others like Mucor and Cryptococcus should be considered. ${ }^{12}$ Marked immune dysregulation in COVID-19 makes system immune becomes impaired, it also harms lung epithelium. ${ }^{3}$ Those dysregulations affect both T-helper cell 2 (Th2) and Th1 responses, including the cytokine release syndrome, which contribute to lung pathology and promote pulmonary microbial proliferation and a subsequent infection. ${ }^{13}$ Decreasing CD4+T and CD8+ T Cells also make COVID-19 patients always in immunosuppression condition. ${ }^{12}$ Moreover, potential routes of secondary infection are medical procedures and invasive technique, thus those patients who had CVC, consumed broad spectrum antibiotics, used steroid, and even had prolonged ICU stays were at risk to get Candida as super infection. ${ }^{3}$

Candidiasis usually appears in ARDS patients. Those who are not developing into ARDS are underestimated and health practitioners often being late in diagnosing. In this case, the patient had moderate COVID-19 but not becoming ARDS patient. She also received steroid and broad-spectrum antibiotics when she had COVID-19 and on admission on day 0, but showed no response to antibiotics. Severely ill/immunocompromised patients have higher probability to suffer invasive mycoses. ${ }^{12}$ Candida are equipped with virulence attributes, enabling them to invade when opportunities arise and cause various infections in humans, especially when the immune system is impaired. ${ }^{14}$ Another study suggested that the sepsis syndrome or septic shock associated with severe COVID-19 may damage the intestinal mucosal barrier, enabling the translocation of concentrated fungus in probiotics $(250-500 \mathrm{mg} /$ day in this case), leading to fungemia. ${ }^{3}$ This patient, in second day of admission, developed into septic shock and showed no better response to antibiotics. Microbial examination was needed and valued to diagnose fungal pneumonia. ${ }^{15}$ The diagnosis of candidiasis and other forms of fungal pneumonia remain challenging, which is mostly due to the low number of yeast cells in circulation or infected tissue. ${ }^{3}$ The sample should be taken with bronchoscopy to keep the hygiene (sterile) and not mixing it with oral fungi like candidiasis oral. Nevertheless, in this case, the patient's condition was not stable to do bronchoscopy and her family did not agree to perform bronchoscopy. Thus, we checked her clinical conditions whether she had oral candidiasis or not. She showed no resolutions for antibacterial drugs, therefore we evaluated her chest $\mathrm{X}$-ray. We also took her sputum and checked from microbial culture. Her sputum culture was positive for non-albicans Candida. Based on the finding, we decided to give her anti-fungal. She was getting better after receiving the treatment and showed less infiltrates in chest X-Ray. It was strong enough to diagnose that she had fungal pneumonia.

Patients who received corticosteroids were also more likely to have superinfection, such as bacterial 
pneumonia or invasive fungal infection than was found in non-corticosteroid groups. ${ }^{16}$ Corticosteroids have immunosuppressive effects on neutrophils, monocytes, and macrophages. It predisposes patients to invasive Candidiasis. ${ }^{3}$ Recent reports indicate an increased rate of fungal infection in COVID-19 patients. Secondary infections also have detrimental effects to the prognosis, thus it should be recognized sooner. COVID-19 patients showed an attenuated monocyte CD80 upregulation and abrogated release of IL-6, TNF, IL1 towards Candida, especially Candida albicans. ${ }^{17}$ Prolonged neutropenia, other immune impairment factors, parenteral nutrition, invasive examination, steroid-used, and broad-spectrum antibacterial drugs are risk factors of Candida infection. ${ }^{12}$ Therefore, if COVID-19 patients develop critically ill condition, it might be the case of secondary infection. ${ }^{17}$

Many studies of hospitalized patients with COVID-19 revealed the patients got empiric antibiotics. However, there is an evidence that the inflammatory serological markers, such as procalcitonin and CReactive Protein (CRP) which are usually associated with bacterial infections, may appear in COVID-19 patients without occuring corresponding bacterial coinfection. Meanwhile, receiving broad spectrum antibiotics can become one of risk factors for developing invasive fungal infection. It should be used wisely and aim for better treatment and more efficient use of equipment, medication, and time. ${ }^{18}$ The application of antibacterial drugs in COVID-19 patients are mainly for two purposes; first is for trial treatment of suspected patients to help differential diagnosis, and second is used to control secondary bacterial infections. Patients who did not achieve resolutions after being given broad spectrum antibiotics could be suspected for fungal infection as secondary infection. Patients with mild COVID-19 who are not treated with glucocorticoid and have no evidence in bacterial infections, in principle, do not need the use of antibacterial drugs. In critically ill patients, prophylactic antibacterial drugs can be used appropriately. ${ }^{19}$ Microbial in sputum must be evaluated, especially in patients who do not go well with antibiotics. Many studies also recommend to systematically screen for fungal pathogens in COVID-19 patients; the sooner the better. ${ }^{12}$

Invasive candidiasis is rare, but has high mortality and needs early recognition and appropriate anti-fungal therapy. ${ }^{12}$ Fungal pneumonia needs attention to be diagnosed. Steroid use and antibacterial drugs should be used appropriately. This patient came to the hospital with dyspnoea and we suspected her of having
ADHF with icterus as complication for liver congestive, but she showed no response for diuretic drugs. Her ECG was normal, her echocardiography was relatively normal with ejection fraction of $76 \%$, and showed no symptoms like JVP elevation, no ronchi and oedema. On her second admission, her condition was getting worse and her tension dropped. We then evaluated the patient's condition and thought about another differential diagnostic like hemaphagocytic lymphohistiocytosis in post COVID-19, but she did not fulfil the criteria. We also gave her broad spectrum antibiotics, but she showed no response. Her culture was negative from bacteria and tuberculosis, therefore we thought about secondary infection like fungal pneumonia and sepsis with complication liver injury. In sepsis, the liver is injured by pathogens, toxins, or inflammatory mediators. The injury progresses from active hepatocellular dysfunction to liver damage and then to liver failure. Liver dysfunction consists of subtle alterations in hepatocellular functions, such as decreased synthesis or decreased clearance function. Liver damage is defined as an irreversible injury to hepatocytes. Liver failure is defined as sustained, severe damage to the liver, and loss of function in $80-90 \%$ of liver cells. Sepsis causes liver injury, and liver injury exacerbates the severity of sepsis, with each amplifying the other's effects. In patients, early manifestations of hepatic dysfunction include elevated serum bilirubin and alkaline phosphatase levels, and the late phase may be marked by elevation of transaminases after prolonged hypotension, which can see in this patient. ${ }^{20}$ That explains why the patient came to the emergency room with one of her major complaints was jaundice.

After concluding all findings, we gave her appropriate anti-fungal treatment and she was getting better. Among patients with septic shock attributed to invasive candidiasis like this patient, the timely administration of anti-fungal therapy is paramount for a favourable outcome and better prognosis. ${ }^{3}$ Source control and exact anti-fungal therapy is needed. The management of invasive candidiasis in patients with COVID-19 is similar to that of non-COVID-19 patients. Echinocandins are the treatment of choice for invasive Candida infections, with fluconazole, liposomal amphotericin B, voriconazole, posaconazole, and isavuconazole being the second line alternatives. Echinocandins are usually well-tolerated and have a favourable pharmacokinetic (PK) profile, with very few drug-drug interactions. ${ }^{3}$ This patient received echinocandins as the first line of anti-fungal in Candida and she felt better afterwards. Study from Haozheng 
Zhang, et al. revealed patients without secondary infection had a significantly higher 60-day discharge rate improvement which is different from patients with secondary infection ( $p<0.001)$ in COVID-19. It is also increasing its mortality and morbidity. ${ }^{21}$

What should be done in the future? Is it a new problem? We should be aware that COVID-19 can develop secondary infection, whether it is bacteria or fungal, even though it is hard to diagnose. It relies on a combination of clinical, radiological, and microbiological factors. ${ }^{9}$ COVID-19 is a risk factor. Nevertheless, there are other risk factors aside from immunosuppression condition due to COVID-19. Steroid use and broad-spectrum antibiotics should be given wisely with consideration of the benefits and the risks. Diagnosis and prompt treatment should be delivered quickly, especially when the patient gets candidiasis as secondary infection. Candidiasis can develop into septic shock and worsen the patient's outcome. Sputum culture should be evaluated for every patient that did not go well with antibiotics treatment. This patient had good outcome and her condition was getting better after we evaluated her condition quickly and gave her anti-fungal treatment. As fungal pneumonia, the individual prognosis is often linked to the severity and outcome of the underlying disease and to whether a reversal of factors affect the patient's immune status. ${ }^{9}$

\section{CONCLUSION}

Post COVID-19 is a condition that should be given more attention, especially when it develops secondary infection and multiorgan damage. Long COVID-19 is a big problem and decreases lung function. Superinfection with fungal pneumonia can worsen the patient's condition, leading to increasing its mortality and morbidity. Candidiasis as fungal pneumonia is hard to diagnose and the mortality is high. It can be seen from this patient, her condition was hard to diagnose and there were many differential diagnoses. It was very challenging because her condition was getting worse if given late diagnosis. Health practitioners should recognize it as soon as possible and give the best treatment for the patients. Further examination should be provided, like microbial culture, especially to those who did not go well with antibiotics. Steroid and antibacterial drugs should be given carefully to patients with COVID-19 as its risk factor for developing secondary infection in the future, with consideration of the risk and the benefits. Therefore, we should be aware with the outcomes of patient with post
COVID-19 syndromes, especially in patients with secondary infections.

\section{ACKNOWLEDGMENTS}

We thanked dr. Susantina (internist and infection consultant in Immanuel Hospital Bandung) and dr. Vika (cardiologist in Immanuel Hospital Bandung) for further identification in this case and useful suggestion. We also thanked Immanuel Hospital Bandung for supporting this case report

\section{REFERENCES}

1. Cennimo DJ. Coronavirus Disease 2019 (COVID19). Medscape https://emedicine.medscape.com/article/2500114overview (2021)

2. Prevention $\mathrm{C}$ for DC and. Post-COVID Conditions. $C D C, \quad$ https://www.cdc.gov/coronavirus/2019ncov/long-term-

effects/index.html?CDC_AA_refVal=https $\% 3 \mathrm{~A} \% 2$ F\%2Fwww.cdc.gov\%2Fcoronavirus\%2F2019ncov\%2Flong-term-effects.html (2021).

3. Arastehfar A, Carvalho A, Nguyen $\mathrm{MH}$, et al. COVID-19-Associated Candidiasis (CAC): An Underestimated Complication in the Absence of Immunological Predispositions? J Fungi (Basel, Switzerland); 6. Epub ahead of print October 2020. DOI: $10.3390 /$ jof6040211.

4. Fernández-de-Las-Peñas C, Palacios-Ceña D, Gómez-Mayordomo V, et al. Defining PostCOVID Symptoms (Post-Acute COVID, Long COVID, Persistent Post-COVID): An Integrative Classification. Int J Environ Res Public Health; 18. Epub ahead of print March 2021. DOI: 10.3390/ijerph18052621.

5. Balasopoulou A, Kokkinos P, Pagoulatos D, et al. Symposium Recent Advances and Challenges in the Management of Retinoblastoma Globe - Saving Treatments. BMC Ophthalmol 2017; 17: 1.

6. Huang I, Pranata R. Lymphopenia in Severe Coronavirus Disease-2019 (COVID-19): Systematic Review and Meta-Analysis. J Intensive Care 2020; 8: 36

7. Zhu X, Lang J. Soluble PD-1 and PD-L1: Predictive and Prognostic Significance in Cancer. Oncotarget 2017; 8: 97671-97682.

8. Vijay S, Bansal N, Rao BK, et al. Secondary Infections in Hospitalized COVID-19 Patients: Indian Experience. Infect Drug Resist 2021; 14: 1893-1903.

9. Mandanas RA. Fungal Pneumonia. Medscape, https://emedicine.medscape.com/article/300341overview (2019).

10. $\mathrm{Du} \mathrm{Y,} \mathrm{Tu} \mathrm{L,} \mathrm{Zhu} \mathrm{P,} \mathrm{et} \mathrm{al.} \mathrm{Clinical} \mathrm{Features} \mathrm{of} 85$ Fatal Cases of COVID-19 from Wuhan. A Retrospective Observational Study. Am J Respir 
Crit Care Med 2020; 201: 1372-1379.

11. Al-Hatmi AMS, Mohsin J, Al-Huraizi A, et al. COVID-19 Associated Invasive Candidiasis. The Journal of Infection 2021; 82: e45-e46.

12. Song G, Liang G, Liu W. Fungal Co-Infections Associated with Global COVID-19 Pandemic: A Clinical and Diagnostic Perspective from China. Mycopathologia 2020; 185: 599-606.

13. Pemán J, Ruiz-Gaitán A, García-Vidal C, et al. Fungal Co-Infection in COVID-19 Patients: Should We be Concerned? Rev Iberoam Micol 2020; 37: 41-46.

14. Rolling T, Hohl TM, Zhai B. Minority Report: The Intestinal Mycobiota in Systemic Infections. Curr Opin Microbiol 2020; 56: 1-6.

15. Zhou P, Liu Z, Chen Y, et al. Bacterial and Fungal Infections in COVID-19 Patients: A Matter of Concern. Infect Control Hosp Epidemiol 2020; 41: 1124-1125.

16. Ni Y-N, Chen G, Sun J, et al. The Effect of
Corticosteroids on Mortality of Patients with Influenza Pneumonia: A Systematic Review and Meta-Analysis. Crit Care 2019; 23: 99.

17. Moser D, Biere K, Han B, et al. COVID-19 Impairs Immune Response to Candida albicans. Front Immunol 2021; 12: 640644.

18. Lansbury L, Lim B, Baskaran V, et al. CoInfections in People with COVID-19: A Systematic Review and Meta-Analysis. J Infect 2020; 81: 266275.

19. Shang Y, Pan C, Yang X, et al. Management of critically ill patients with COVID-19 in ICU: statement from front-line intensive care experts in Wuhan, China. Ann Intensive Care 2020; 10: 1-24.

20. Yan J, Li S, Li S. The Role of the Liver in Sepsis. Int Rev Immunol 2014; 33: 498-510.

21. Zhang H, Zhang Y, Wu J, et al. Risks and Features of Secondary Infections in Severe and Critical Ill COVID-19 Patients. Emerg Microbes Infect 2020; 9: 1958-1964. 